\title{
Designing a Quenching System for Grinding Steel Balls - Case Study
}

\author{
Loice Keresia Gudukeya , Charles Mbohwa \\ University of Johannesburg, Faculty of Engineering and the Built Environment, Auckland Park 2006, P.O Box 524, Johannesburg, South \\ Africa
}

\begin{abstract}
The availability of grinding balls of superior quality than those produced at a Harare company in Zimbabwe, resulted in loss of business. In order to retain business, a new ball that has a similar chemical composition to that of imported grinding balls was developed. Proper thermo-mechanical treatment has to be done after casting, in order for the ball to be of satisfactory quality. In this research, heat treatment tests were done on the developed ball to determine a suitable quenching medium for the heat treatment cycle. Using the results of the heat treatment tests obtained, a suitable heat treatment cycle which utilises optimum quenching medium and has effective agitation for minimal defects was designed.
\end{abstract}

\section{Introduction}

A Harare company's core business is the manufacturing of grinding steel balls used in mining operations. Cast steel balls are used to grind run of mine coarse materials in a ball mill. The balls vary in size from $15 \mathrm{~mm}$ to $120 \mathrm{~mm}$ in in diameter and are manufactured from scrap metal. However, the company now faces stiff competition from imports of the same product that is coming in with better quality. There is a need to counter this competition by producing steel balls that are of at least the same quality or better. The chemical composition of a locally developed steel ball is already in place. What is required is the heat treatment cycle which include quenching and tempering process ideal for the developed ball to give the desired results.

\section{Literature review}

\subsection{Quenching}

Quenching is the process of cooling a metal at a rapid rate from a predetermined high temperature. This process is important as it is key in addressing the main challenge of manufacturing steel balls of high quality. When hardening metallic materials, cooling is an important and decisive element in determining the properties of the part such as it hardness. [3]

\subsection{The quenching process}

When heat treatment fluids are used to quench metals, cooling occurs in three distinct stages: film boiling, nucleate boiling and convective heat transfer.[1]
Film Boiling - Film boiling, also known as the "vapor blanket" stage, occurs upon initial immersion. Contact between the hot metal surface and quenchant creates a layer of vapor (known as the Leidenfrost phenomenon) due to the supply of heat being greater than that which is carried off. The stability of the vapor layer, and thus the ability of the oil to harden steel, is dependent on the metal's surface irregularities, oxides present, surfacewetting additives (which accelerate wetting and destabilize the layer), and the quench oil's molecular composition (including the presence of more volatile oil degradation by-products). Cooling in this stage is a function of conduction through the vapor envelope and is relatively slow since the vapor blanket acts as an insulator.

Nucleate Boiling - As the part cools, the vapor blanket collapses at points and nucleate boiling (violent boiling of the quenchant) results. Heat transfer is fastest during this stage, with heat transfer coefficients sometimes over two orders of magnitude higher than during film boiling, largely due to the heat of vaporization.

Convective Heat Transfer - When the part has cooled below the boiling point of the quenchant, slow cooling occurs by convection and conduction (also called the "liquid" stage). Cooling rate during this stage is slow, and is exponentially dependent on the oil's viscosity, which varies with the degree of oil decomposition. Heat-transfer rates increase with lower viscosities and decrease with increasing viscosity.

The ideal quenchant is one that exhibits little or no vapour stage, a rapid nucleated boiling stage, and a slow rate during convective cooling. [4]

\subsubsection{Quench cracks}


Cracking may happen during quenching or sometimes after quenching. The causes of cracking are [2]:

- Excessive amount of non-metallic inclusions in the steel

- Quenching from a high temperature - high austenitizing temperatures lead to grain coarsening of austenite resulting in fine grained, highly dislocated martensitic structure which is more likely to crack.

- Improper selection of quenching medium - By using a quenching fluid which gives higher cooling rate than the one necessary may lead to cracking.

- Uneven quenching in the quenching bath

- Improper entry of the component into the quenching media causing eccentric and nonuniform cooling

Therefore the selection of quenching medium, the design of the quench tank as well as agitation system has to be done carefully in order to prevent or minimise quench cracks.

\subsection{Heat treatment test}

\subsubsection{Materials}

The materials used in the experiments are: the steel balls obtained from the company (its chemical composition is shown in Table 1); quenching oil sourced from Jacob Bethel Corporation.

Table 1: Chemical Composition of the ball by percentage

\begin{tabular}{lllllllll}
\hline $\mathrm{C}$ & $\mathrm{Si}$ & $\mathrm{Mn}$ & $\mathrm{P}$ & $\mathrm{S}$ & $\mathrm{Cr}$ & $\mathrm{Ni}$ & $\mathrm{Cu}$ & $\mathrm{Fe}$ \\
\hline 2.4 & 0.8 & 0.39 & 0.02 & 0.02 & 18.8 & 0.26 & 0.13 & 76. \\
2 & 5 & 7 & 6 & 8 & 7 & 5 & 3 & 9 \\
\hline
\end{tabular}

\section{Equipment}

The equipment used in the experimental procedure include:

- Heat treatment furnace

- Hardness test machine

- Tongs

- Grinding media

- Oil Quench Tank

\subsubsection{Procedure}

1. Two samples were cut from the steel grinding ball. Both samples were heated in a heat treatment furnace to $10000 \mathrm{C}$ for 4 hours.

2. One sample was quenched in an oil bath of Castrol HT 5 heat transfer fluid and the other was quenched in plain water.
3. The hardness of the samples were then measured using the Rockwell hardness test. A preliminary test force is first applied to a sample using a diamond indenter. This load represents the zero or reference position that breaks through the surface to reduce the effects of surface finish. After the preload, an additional load, call the major load, is applied to reach the total required test load. This force is held for a predetermined amount of time to allow for elastic recovery. This major load is then released and the final position is measured against the position derived from the preload, the indentation depth variance between the preload value and major load value. This distance is converted to a hardness number.

4. The samples were returned into the furnace for tempering at $2500 \mathrm{C}$ and held at this temperature for one and half hours. Tempering is done to relieve the metal of any residual stresses that might be set up during the quenching process, thereby reducing the likelihood of quench cracks developing in the metal. The tempering process also has the effect of softening the surface of the metal.

5. The samples were taken out of the furnace and left to cool in open air for an hour.

\subsubsection{Design procedure}

Data collection

Before the design of the system, data pertaining to the design parameters was gathered. This data was used in the sizing and selection of various equipment of the system. This data included capacity of the system, desired daily output, temperature parameters for the quench oil and properties of the quench oil.

\section{Evaluation of cooling methods}

After determining the quenchant to be used and collecting data pertaining to design parameters, three cooling methods of the quenchant were evaluated. Quench bath temperature control is of great importance in that it is the final parameter determining the mechanical properties of the grinding balls. The methods were then evaluated using a selection matrix table, where predetermined parameters are given different weights.

\section{System design}

All the data collected as well as the chosen method of cooling were then used in the system design. In the design, the sizing of the equipment such as the heat exchanger and pumps was done.

\section{Results and discussion}

After the heating and soaking, the samples were quenched in water and oil separately. This was done to compare the efficiency of oil against water. The hardness tests were taken using the Rockwell hardness test. The results of the hardness values taken were recorded in Table 2 . 
Table 2: Hardness test results

\begin{tabular}{lcc}
\hline & $\begin{array}{c}\text { Water quenched } \\
\text { sample/HRC }\end{array}$ & $\begin{array}{c}\text { Oil quenched } \\
\text { sample/HRC }\end{array}$ \\
\hline First batch & 64 & 62 \\
Second batch & 65 & 63 \\
Third batch & 64 & 63 \\
Average & 64.3 & 62.7 \\
hardness & & \\
\hline
\end{tabular}

The results of these tests were then used to determine the quench fluid for the system. The resulting hardness values obtained after the Rockwell hardness test was done on both samples and are presented in Table 2. The water quenched sample shown in Figure 1 gave a higher hardness value possibly linked to the fact that water has a lower viscosity than heat transfer of oil and also that the initial temperature of water was lower than that of the oil.

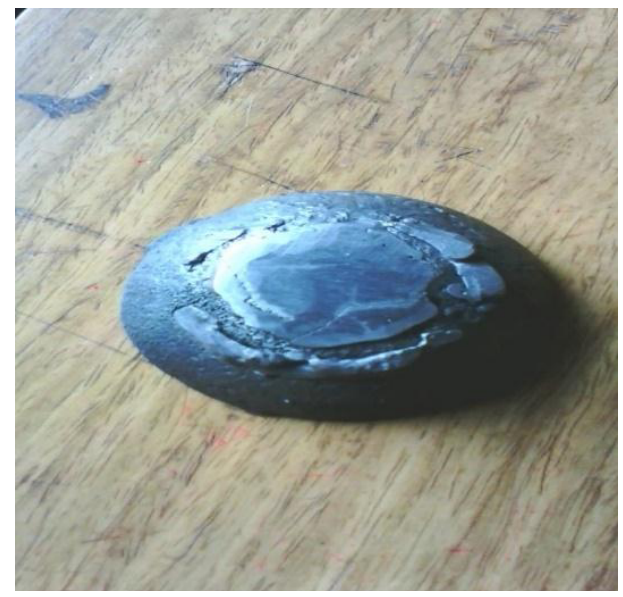

Figure 1: Water quenched sample showing quench cracks

The water quenched sample also had quench cracks which may be attributed to the high heating temperature which may be unsuitable for quenching in water. During quenching, water has a tendency to cause vapour entrapment on the surface of the metal causing uneven hardness and therefore unfavourable stress distribution. This uneven stress distribution may be responsible for the cause of cracks which occur as residual stress in the metal. This is subsequently relieved by tempering in order to get a more even stress distribution throughout the metal.

Oil is more viscous than water, thereby producing a sample with improved toughness and ductility. Oil has a slower cooling rate than water, which means that there is greater depth of hardness and less residual stress in the ball. Slower cooling rate also means that there is a more even stress distribution in the sample, which explains why the sample quenched in oil did not develop cracks. Although hardness value obtained after quenching in oil is lower than that obtained after quenching in water, it also meets the target hardness of at least $60 \mathrm{HRC}$. Hence Castrol HT 5 Heat Transfer oil was found to be the most suitable quenching medium and will be adopted for use in the quenching system.
From the experiments conducted, the quenching system will consist of the following components:

- Main quench tank (Size)

- Quench tank components such as draft tubes, marine impeller agitators, heaters and oil fume hood.

- Oil storage tank

- Heat exchanger

- Pumps which will circulate the quenchant

This design covers the Sizing of the Quench tank, Rotation of balls, Sizing of the agitators, Design of gearbox for agitators, Draft tube design, Quench Tank Layout, Sizing of the heat exchanger, Sizing of the pump to heat exchanger, Sizing of the pump from storage tank to quench tank, sizing of Heaters and Filtration Equipment. Figures2, 3 and 4 show the top view of the quench tank, the side view of the quench tank and the system layout respectively.

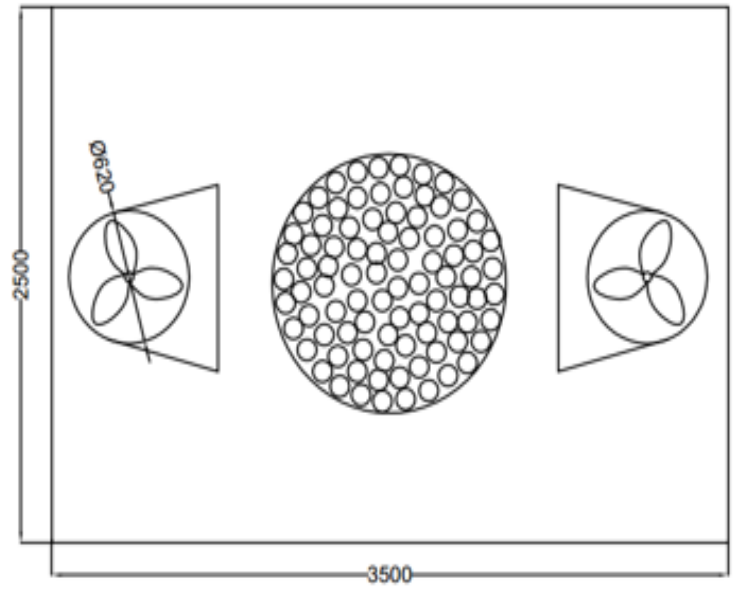

Figure 2: Top view of the quench tank

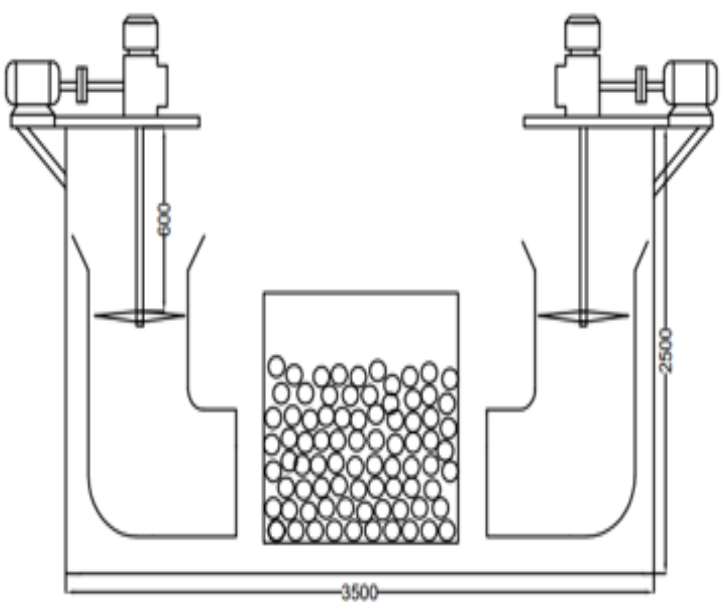

Figure 3: Side view of the quench tank

\section{Quench system design}




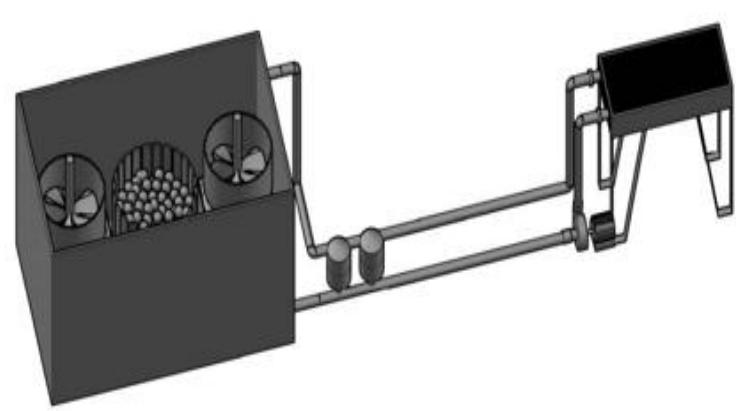

Figure 4: System layout

\section{Conclusion and recommendations}

The uniform quenching achieved results in a minimum number of rejected parts. The mathematical calculations related to the sizing of the equipment as well as design of the fluid flow dynamics in the quench tank show that the grinding balls quenched using this system will be of comparable quality with that of imported balls. An accurate cost benefit analysis of the project will need to be done versus the expected growth in demand for the new ball. Purchasing of the oil will be part of running costs therefore ways of maintaining the oil need to be established in order to increase its running life. These may include regularly checking for sludge content and moisture content as these contaminants may affect the quench uniformity if present in the oil in large quantities. Accelerants may be added to the oil in order to prolong its life in use.

\section{References}

1. CE Bates, G. T. N. C., 1993. Handbook of Quenchants and Quenching Technology. Ohio, USA: ASM International.

2. Kumar, S. S., 1999. Material and Murtallurgical Science. 1st ed. s.l.:Anuradha Agencies.

3. Segerbag S, 2001. Industrial cooling - some problems and their solutions. IVF industrial research and development corporation Gothenburg Sweden.

4. A. A. Yekinni, S. K. Bello, L. O. Mudashiru, L. A. Animashaun. 2015. Modification and Fabrication of Quenching Machine: Influence of Agitation and Temperature Gradient on Hardness of Al 6050 Alloy. International Journal of Materials Engineering. 